\title{
Regional brain activation/deactivation during word generation in schizophrenia: fMRI study
}

John P. John, Harsha N. Halahalli, Mandapati K. Vasudev, Peruvumba N. Jayakumar and Sanjeev Jain

\section{Background}

Examination of the brain regions that show aberrant activations and/or deactivations during semantic word generation could pave the way for a better understanding of the neurobiology of cognitive dysfunction in schizophrenia.

\begin{abstract}
Aims
To examine the pattern of functional magnetic resonance imaging blood oxygen level dependent activations and deactivations during semantic word generation in schizophrenia.

Method

Functional magnetic resonance imaging was performed on 24 participants with schizophrenia and 24 matched healthy controls during an overt, paced, 'semantic category word generation' condition and a baseline 'word repetition' condition that modelled all the lead-in/associated processes involved in the performance of the generation task.
\end{abstract}

\section{Results}

The brain regions activated during word generation in healthy individuals were replicated with minimal redundancies in participants with schizophrenia. The individuals with schizophrenia showed additional activations of temporo-parieto-occipital cortical regions as well as subcortical regions, despite significantly poorer behavioural performance than the healthy participants. Importantly, the extensive deactivations in other brain regions during word generation in healthy individuals could not be replicated in those with schizophrenia.

\section{Conclusions}

More widespread activations and deficient deactivations in the poorly performing participants with schizophrenia may reflect an inability to inhibit competing cognitive processes, which in turn could constitute the core informationprocessing deficit underlying impaired word generation in schizophrenia.

\section{Declaration of interest}

None.
Schizophrenia has been unequivocally shown to be associated with impaired verbal fluency. ${ }^{1}$ However, functional imaging studies that have examined the aberrant brain activations ${ }^{2}$ and deactivations ${ }^{3}$ underlying impaired verbal fluency in schizophrenia have yielded inconsistent results. Studies that have examined the brain regions associated with word generation in healthy participants have also generated inconsistent findings. ${ }^{4,5}$ The heterogeneity among earlier studies with regard to their usage of phonological or semantic tasks, covert or overt paradigms, paced or unpaced trials, nature of the baseline task with respect to whether it models all the lead-in/associated processes involved in word generation, volume acquisition methods, sample sizes and statistical thresholds used for making inferences may have resulted in these inconsistent findings.

Impaired semantic verbal fluency has been suggested to be the best candidate cognitive endophenotype of schizophrenia. ${ }^{1}$ Semantic verbal fluency has been shown to have higher discriminating power to elicit significantly larger group differences between individuals with schizophrenia and healthy controls than phonological fluency. ${ }^{6}$ There are previous reports of paced, overt semantic fluency paradigms being more useful for functional magnetic resonance imaging (fMRI) experiments, given their superiority with regard to control over and monitoring of behavioural responses. ${ }^{7}$ Additionally, clustered volume acquisition allows for the stimulus presentation and overt response to occur during the 'silent' periods between image acquisition, thereby making the 'online' measurement of behavioural performance possible. $^{8,9}$ In a previous study that employed an overt, paced, semantic verbal fluency paradigm with a clustered volume acquisition, Ragland et $a l^{10}$ reported greater activations in participants with schizophrenia than controls during semantic word generation, indicating an inefficient brain oxygen level dependent (BOLD) haemodynamic response.
In the present study, we recorded BOLD haemodynamic responses using a clustered fMRI acquisition paradigm while participants with schizophrenia (the schizophrenia group) and matched healthy controls (control group) performed an experimental protocol that included alternating word generation and word repetition blocks. An overt, paced, visually cued semantic category word generation task with clustered volume acquisition was used as the experimental paradigm. Since a 'resting' baseline condition is incapable of modelling all the lead-in/associated processes involved in word generation, we chose an overt visually cued 'word repetition task' as the baseline condition to obtain the word generation $v$. word repetition contrast to reflect activations during word generation. Since all the lead-in/associated cognitive and motor processes involved in word generation (specifically, visual word form recognition and vocalisation of verbal responses) were modelled in the word repetition task, it was expected that there would be no brain regions that are more active during the less cognitively demanding word repetition task in comparison with the more cognitively demanding word generation task. Therefore, the BOLD signals, if any, which would be observed in the word repetition $v$. word generation contrast reflect the 'deactivations' in those regions during word generation. Thus, an optimised experimental design was arrived at a priori, employing which, we expected to make reliable and valid inferences regarding the brain regions that are activated and deactivated during semantic category word generation and their dysfunction in schizophrenia. We aimed at generating population-level inferences regarding activations and deactivations in both the control and the schizophrenia group during word generation, by performing separate one-sample random-effects analyses for each group, of the word generation $v$. word repetition and word repetition $v$. word generation contrasts respectively. We hypothesised that there would be 
significant differences in the pattern of BOLD activations/ deactivations in the poorly performing participants with schizophrenia, in comparison with the healthy controls. We further aimed to link response latencies as an index of behavioural performance with activations/deactivations using regression analysis and expected to find significant correlations.

\section{Method}

The study was carried out at the National Institute of Mental Health and Neurosciences (NIMHANS), Bangalore, India, with approval from the NIMHANS Ethics Committee, thus conforming to the ethical standards laid down in the 1964 Declaration of Helskinki. Written informed consent was obtained from all participants (and their legally qualified representatives in the case of individuals with schizophrenia) prior to enrolling them into the study.

\section{Participants}

The study samples comprised 24 healthy participants (age: 19-44 years, mean 27.30 years; 19 males), who were recruited by word of mouth, and 24 individuals with schizophrenia (age: 17-45 years, mean 27.65 years, 16 males), recruited from the out-patient department of NIMHANS by purposive sampling. The samples were matched for age and education status. The sociodemographic and clinical characteristics of the samples are given in Table 1. Only right-handed participants (as determined by modified Annett's inventory $)^{11}$ in the age group 17 to 50, with a Mini-Mental State Examination (MMSE) ${ }^{12}$ score of 23 or more were included in the study. A score of 23 or above was set as an inclusion criterion to ensure that all those in the schizophrenia group who were recruited into the study had an adequate level of overall cognitive functioning, ${ }^{12}$ so that the verbal fluency deficits, if any, would not be accounted for by general cognitive dysfunction. All participants were native speakers of South Indian Dravidian languages and were comfortable in reading their preferred language. None of these participants were formally exposed to a language other than their mother tongue, prior to 6 years of age. The matrix reasoning module of Wechsler's Adult
Intelligence Scale-III (WAIS-III) ${ }^{13}$ was administered to all participants to obtain an estimate of their perceptual organisation index as a reflection of their intellectual abilities, independent of verbal abilities.

The diagnosis of schizophrenia or schizophreniform disorder was arrived at using criteria from DSM-IV ${ }^{14}$ based on the consensus of a research psychiatrist who conducted a semistructured interview and a trained research assistant who used the Mini International Neuropsychiatric Interview (MINI) Plus. ${ }^{15}$ In total 15 of the 24 individuals in the schizophrenia group were on medications at the time of fMRI acquisition, whereas the remaining 9 were antipsychotic-naive/free. The antipsychotic doses were expressed as risperidone equivalents according to Woods ${ }^{16}$ to be entered as covariates in the BOLD group comparisons, since antipsychotic drugs could potentially increase cerebral blood flow to the prefrontal cortex ${ }^{17,18}$ and the basal ganglia, ${ }^{19}$ as discussed subsequently. The medication status of the participants with schizophrenia are given in Table 1 (details of medication are given in Table DS1). Baseline severity of schizophrenia psychopathology was evaluated using the Positive and Negative Syndrome Scale (PANSS) ${ }^{20}$ for all participants by two trained raters who had established good interrater reliability. Individuals in the control group were ascertained to be free from Axis I or II psychiatric disorders using the MINI-Plus. ${ }^{15}$ The presence of any other medical/neurological condition requiring continuous medications, current psychotropic use and history of psychiatric illness in first-degree relatives were ruled out by an unstructured clinical interview.

\section{Experimental design}

The fMRI experiment consisted of two tasks: the 'semantic category word generation' condition and the 'word repetition' condition administered alternately in six blocks each as a paced, overt and visually cued paradigm, implemented using the E-Prime stimulus presentation software, version 2.0 (PST Inc, Philadelphia, USA, www.pstnet.com/) operating on Windows, within the Integrated Functional Imaging System (IFIS-SA) (Invivo, Orlando, Florida, USA, www.invivocorp.com). All participants were briefed about the task $1-3 \mathrm{~h}$ prior to the imaging session,

\section{Table 1 Sociodemographic and clinical characteristics of the study samples}

\begin{tabular}{|c|c|c|}
\hline & \multicolumn{2}{|c|}{ Participants $(n=48)$} \\
\hline & $\begin{array}{l}\text { Control group } \\
\qquad(n=24)\end{array}$ & $\begin{array}{l}\text { Schizophrenia group } \\
\qquad(n=24)\end{array}$ \\
\hline \multicolumn{3}{|l|}{ Gender, $n(\%)$} \\
\hline Male & $19(79.20)$ & $16(66.70)$ \\
\hline Female & $5(20.80)$ & $8(33.30)$ \\
\hline Age, years: mean (s.d.) range & $27.42(6.27)$ 19-44 & 30.13 (9.48) 17-47 \\
\hline \multicolumn{3}{|l|}{ Education } \\
\hline Formal education, years: mean (s.d.) range & $13.50(2.27) 10-17$ & $12.04(3.10) 6-18$ \\
\hline \multicolumn{3}{|l|}{ Diagnosis, $n$} \\
\hline Paranoid schizophrenia & & 19 \\
\hline Undifferentiated schizophrenia & & 5 \\
\hline Age at onset of illness, years: mean (s.d.) range & & $29.75(9.69)$ 15-46 \\
\hline Duration of illness, months: mean (s.d.) range & & $30.78(17.85) 3-60$ \\
\hline \multicolumn{3}{|l|}{ Medication status } \\
\hline Antipsychotic naive/free, $n$ & & $6 / 3$ \\
\hline On medication, $n$ & & 15 \\
\hline Risperidone dose (mg/day), mean (s.d.) & & $2.46(1.30)$ \\
\hline \multicolumn{3}{|c|}{ Positive and Negative Syndrome Scale (PANSS) mean (s.d.) } \\
\hline Positive score & & $13.96(6.03)$ \\
\hline Negative score & & $15.29(7.36)$ \\
\hline Global psychopathology score & & $25.21(7.62)$ \\
\hline PANSS, total & & $54.50(16.45)$ \\
\hline
\end{tabular}


when they practised a short four-block version of the task (two blocks each for word repetition and word generation), comprising categories other than those included in the imaging session ('wooden articles' and 'kitchen articles'). Each block in the fMRI experiment was preceded by the display of an instruction slide for $8 \mathrm{~s}$ that specified the category ('word repetition' or 'word generation') and reminded the participants to say 'pass' if they could not think of an appropriate example in the case of word generation blocks. Each block comprised seven trials of either word repetition or word generation. The word repetition blocks that served as the baseline, involved repeating the cue word 'pass' as soon as it appeared on the screen. For the word generation blocks, participants were instructed to overtly produce an exemplar of the category word-cue that was displayed on the screen (Fig. 1(a)). The category cue words used for the word generation trials were 'animals', 'vegetables', 'birds', 'fruits', 'flowers' and 'trees'. Participants were instructed to keep their gaze fixed on the centre of the screen and to respond with unique examples from the category as soon as the category-word cue was presented. In order to minimise across-block differences in vocalisation responses according to the number of exemplars produced, participants were instructed to say 'pass' if they could not think of an appropriate example. The timeline of each trial allowed the verbal responses to be made during the 'silent period' (2500 ms) of the compressed echoplanar imaging (EPI) sequence with a repetition time (TR) of $4 \mathrm{~s}$ (Fig. 1(b)).

All stimuli were in white over a black background and measured $2.5 \mathrm{~cm}$ vertically on the screen subtending a visual angle of about $2.4^{\circ}$ and were displayed using a fibre optic, liquid crystal display-based video system controlled by the IFIS-SA system. The responses that were picked up by a magnetic resonance compatible microphone integrated within the projection hood, were recorded onto a computer hard disk using Microsoft Windows Media Encoder and played back later for scoring as 'correct', 'incorrect', 'repetitive', 'pass' or 'no response'. The verbal fluency correct responses (VFCR) and the verbal fluency total responses (VFTR: correct + incorrect + repetitions + 'pass' responses) across the six word generation blocks were calculated and used as covariates in the fMRI analysis to adjust for performance differences. Response latency for each trial was measured as the time interval from the presentation of the visual cue-word to the onset of the verbal response. Latency measurements were not possible in three participants in each group for technical reasons. Mean latencies for all the responses in the word repetition trials and the word generation trials were calculated for each of the remaining 42 participants.

\section{Image acquisition}

Magnetic resonance images were acquired on a Philips Achieva 3.0 T scanner using a SENSE-8 head coil. Single-shot echoplanar images were acquired at a resolution of $2 \times 2 \times 6 \times \mathrm{mm}$ (repetition time (TR): $4000 \mathrm{~ms}$; echo time (TE): $35 \mathrm{~ms}$; flip angle: $90^{\circ}$; EPI factor: 59) with a total scan time of $7 \mathrm{~min} 28 \mathrm{~s}$ for acquisition of 108 volumes. The initial four functional volumes were discarded to allow the fMRI signal to reach steady state. Image acquisition was completed within the first $1.5 \mathrm{~s}$ of each TR, providing a $2.5 \mathrm{~s}$ 'silent' period that was used for noise-free collection of verbal responses. Head movements were minimised by applying a band over the forehead. $T_{1}$-weighted structural images with a resolution of $1 \times 1 \times 1 \mathrm{~mm}$ were also acquired in the same imaging session using a magnetisation prepared rapid acquisition gradient echo (MPRAGE) sequence $\left(\mathrm{TR} / \mathrm{TE}=8.2 / 3.8 \mathrm{~ms}\right.$; flip angle: $8^{\circ}$; sense factor: 3.5$)$.

\section{Image analysis}

Preprocessing of the EPI images and further analyses were performed using SPM5 (www.fil.ion.ucl.ac.uk/spm/software/ spm5/) running on MATLAB (MathWorks Inc, Natick, Maryland, USA, www.mathworks.com/) operating on Windows XP. Briefly, the functional images of each participant were realigned with the first image to reduce movement-related artefacts. The 'unwarp' tool of SPM5 was then employed to minimise the geometrical distortion at the air-tissue interfaces, which is compounded by movement (susceptibility-by-movement interaction). The images were then coregistered with the $T_{1}$-weighted structural images using a rigid body transformation and normalised to the standard MNI space using the ICBM152 template, following which they were smoothed by convolving with a Gaussian kernel of $9 \mathrm{~mm}$ full-width at half maximum.

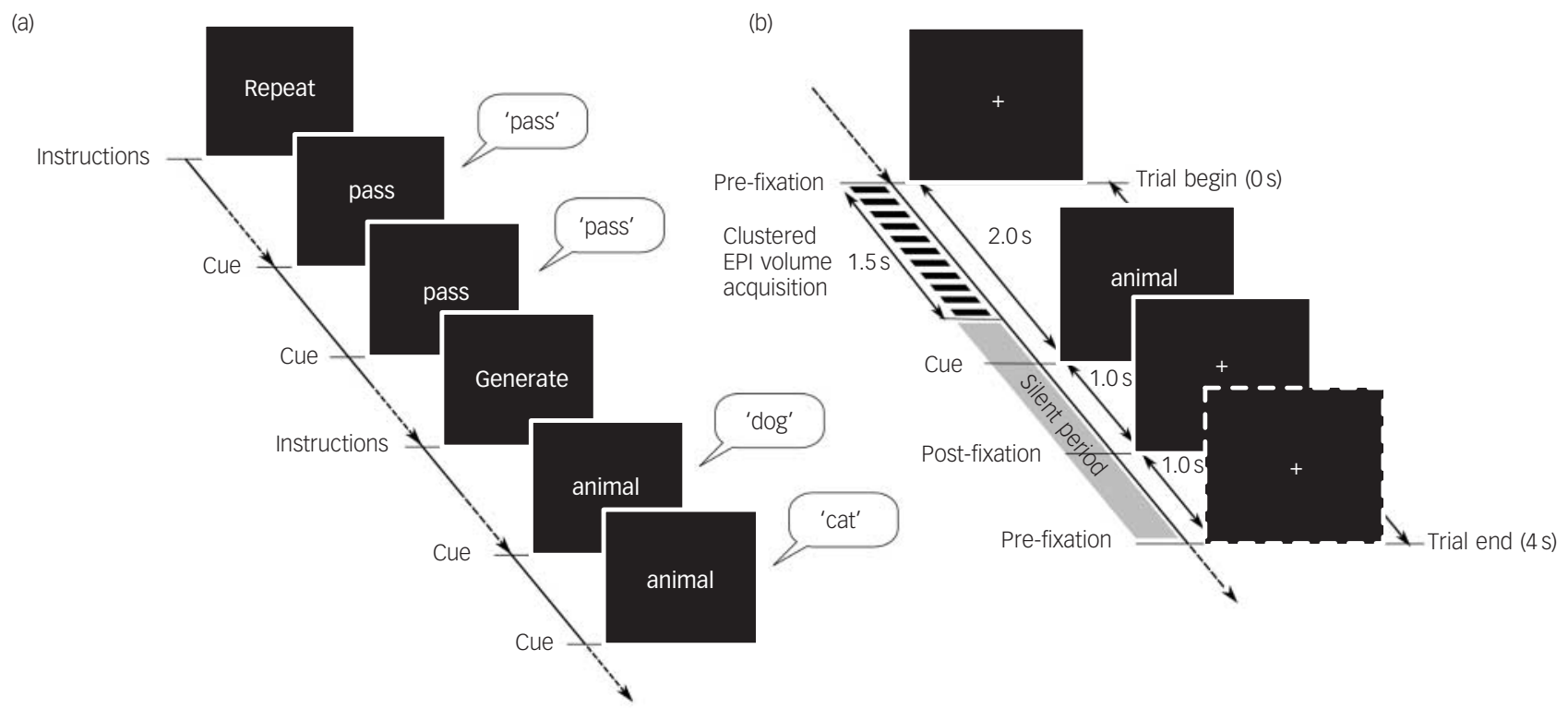

Fig. 1 Verbal fluency task design. 
For each participant, the maximum incremental movement between any two successive scans during the run in each of the three translational planes $(X, Y, Z)$ and rotational axes (pitch, roll, yaw) was estimated from the parameters obtained during the realignment process. The maximum incremental translational movement was less than $2 \mathrm{~mm}$ and rotational movement less than $0.09^{\circ}$ for all participants except for one from the schizophrenia group ( $2.45 \mathrm{~mm}$ in the $Z$-plane). A Mann-Whitney $U$-test showed no significant between-group differences in any of the six parameters (Table DS2).

A general linear model (GLM) was then specified with two conditions of interest - 'word generation' and 'word repetition'. The presentation of instructions between blocks was modelled separately. These task-related regressors were convolved with the canonical haemodynamic response function in SPM5. Realignment parameters generated in the realignment step were included as additional regressors. A high-pass filter with a cut-off of $144 \mathrm{~s}$ was used to filter slow signal drifts. The GLM parameters were estimated using a restricted maximum likelihood algorithm, implemented in SPM5 using a summary statistics approach, and queried using a T-contrast for word generation $v$. word repetition and word repetition $v$. word generation to produce statistical parametric maps (SPMs). The contrast images generated for individual participants (see Fig. DS1, for the design matrix) from each group were then entered into a one-sample random-effects analysis model separately for individuals in the control and schizophrenia groups, with age, gender, education, score on the matrix reasoning test of WAIS-III, VFCR, VFTR, antipsychotic medication dosage (risperidone equivalents) and anticholinergic dosage (trihexyphenidyl dosage) as covariates (see Fig. DS2, for the design matrix) to identify areas of significant activation and deactivation associated with word generation. Since SPM uses a mass univariate approach, correction for multiple comparisons was applied by employing false discovery rate estimations with the level of significance set a priori at false discovery rate $P<0.05$. In addition, an extent threshold of 20 voxels was used while generating the statistical parametric maps. A post hoc between-group comparison was then carried out using the Statistical Non-Parametric Mapping (SnPM; University of Michigan Biostatistics Department, Michigan, USA, www.sph. umich.edu/ni-stat/SnPM) toolbox within SPM5. The SnPM toolbox uses a non-parametric permutations-based statistical approach to voxel-wise analysis of neuroimaging data and is free from the more restrictive assumption of the parametric approaches with regard to the data having to be derived from strictly homogeneous discrete Gaussian random fields with a stationary covariance structure. ${ }^{21}$

Finally, a regression analysis to confirm the relationship between task-related activations/deactivations and behavioural performance was performed on the word generation $v$. word repetition and word repetition $v$. word generation contrast images respectively, with the difference in mean latencies between word generation and word repetition trials ( $\operatorname{lat}_{\text {gen }}-\mathrm{lat}_{\mathrm{rep}}$ ) as the regressor of interest. The same covariates included in the onesample random-effects analyses were also entered in the regression analyses (see Fig. DS3 for the design matrix). Coordinates of brain regions reported here were converted from the Montreal Neurological Institute (MNI) space to the Talairach space using the mni2tal algorithm (http://imaging.mrc-cbu.cam.ac.uk/ imaging/MniTalairach), operating on Windows XP. Anatomical Brodmann area labels were generated for the transformed coordinates using Talairach Client (Version 2.4.2), ${ }^{22}$ also on Windows XP.

\section{Results}

\section{Analysis of task performance during scanning}

The VFTR and VFCR scores were calculated to assess the behavioural performance in the word generation task (Table 2). Both VFTR and VFCR were significantly lower in the schizophrenia group compared with the control group (VFTR: $U=85.5, P<0.001$; VFCR: $U=81.00, P<0.001)$. Both groups of participants displayed a high degree of accuracy in the baseline word repetition condition (control group 100\%; schizophrenia group $98.4 \%$ ).

\section{BOLD activations associated with word generation in control and schizophrenia groups}

The brain regions showing significant BOLD activations as revealed by one-sample random-effects analysis (contrast: word generation $v$. word repetition; false discovery rate $P<0.05$ with 20 voxels extent threshold) in the control and schizophrenia groups are given in Tables DS3 and DS4 respectively. The $t$-maps of the above analyses are provided in Figs 2 and DS4 (design matrices for the participant-level contrasts and one-sample random-effects are given as Fig. DS1 and DS2).

One-sample random-effects analysis of the word generation $v$. word repetition contrast revealed that activations were seen in the control group in inferior frontal gyrus (Brodmann area 47/46), anterior cingulate cortex (Brodmann area 32), rostral prefrontal cortex (Brodmann area 10), medial frontal cortex (Brodmann area $6,8,9$ ), insula (Brodmann area 13) and thalamus bilaterally as well as middle frontal gyrus (Brodmann area $6,8,9$ ), precentral gyrus (Brodmann area 6) and caudate in the left hemisphere. Except for the left caudate and bilateral thalamus, the activations in all the above brain regions were replicated in the schizophrenia group. Participants with schizophrenia, however, showed additional activations in multiple other brain regions, including bilateral superior temporal gyrus, left-sided middle temporal gyrus, inferior parietal lobule, fusiform gyrus, angular gyrus, lingual gyrus, cuneus and red nucleus as well as right-sided middle

\begin{tabular}{|c|c|c|c|c|c|c|}
\hline & \multicolumn{2}{|c|}{ Control group $(n=24)$} & \multicolumn{2}{|c|}{ Schizophrenia group $(n=24)$} & \multirow[b]{2}{*}{$\begin{array}{c}\text { Mann-Whitney } \\
\text { U-test }\end{array}$} & \multirow[b]{2}{*}{$P$} \\
\hline & Mean (s.d.) & $\begin{array}{l}\text { Percentage of total } \\
\text { number of trials }\end{array}$ & Mean (s.d.) & $\begin{array}{c}\text { Percentage of total } \\
\text { number of trials }\end{array}$ & & \\
\hline Total verbal responses & 40.08 (1.93) & 95 & $34.54(4.52)$ & 82 & 85.5 & $<0.001$ \\
\hline Correct responses & $36.25(3.96)$ & 86 & $29.38(5.77)$ & 70 & 81.00 & $<0.001$ \\
\hline Incorrect responses & $0.08(0.41)$ & $<1$ & $0.22(1.04)$ & $<1$ & & \\
\hline Repetitive responses & $0.25(0.53)$ & $<1$ & $0.65(1.11)$ & $<1$ & & \\
\hline Pass responses & $3.50(3.56)$ & 8 & $4.30(6.32)$ & 10 & & \\
\hline No responses & $1.88(1.96)$ & 5 & $7.43(4.62)$ & 18 & 83.5 & $<0.001$ \\
\hline
\end{tabular}


(a)

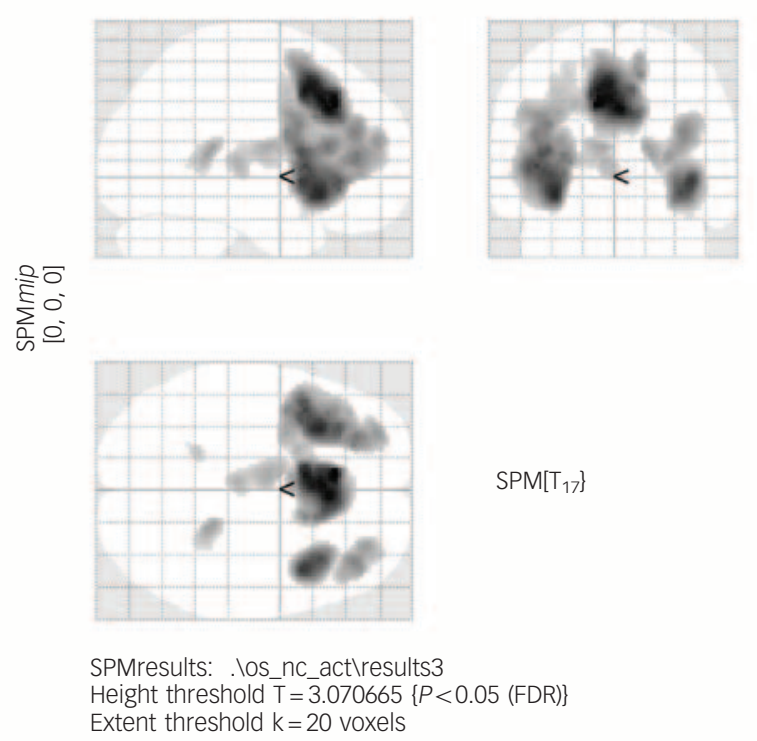

(b)
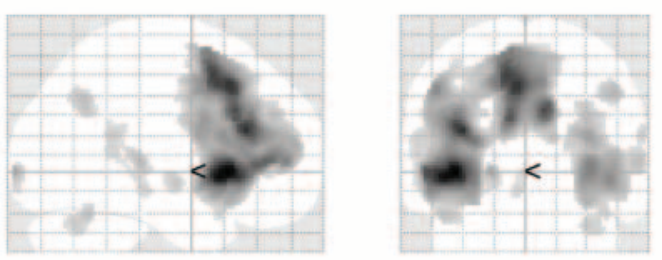

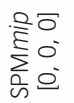

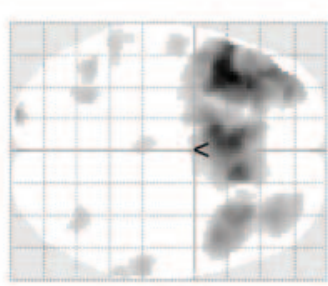

SPMresults: . Ios_sz_act 1 results5

Height threshold $\overline{\mathrm{T}}=2.957044\{P<0.05($ FDR $)\}$

Extent threshold $k=20$ voxels

\section{Fig. 2 One-sample random-effects analysis, word generation-word repetition contrast.}

(a) Blood oxygen level dependent (BOLD) functional magnetic resonance imaging (fMRI) signal responses in the control group ( $n=24,1$ condition, 6 covariates, leaving 17 degrees of (a) Blood oxygen level dependent (BOLD) functional magnetic resonance imaging (fMRI) signal responses in the control group ( $(n=24,1$ condition, 6 covariates, leaving 17 degrees of
freedom); (b) BOLD fMRI signal responses in the schizophrenia group $(n=24,1$ condition, 8 covariates, leaving 17 degrees of freedom); $t$-maps thresholded at $P<0.05$, corrected for

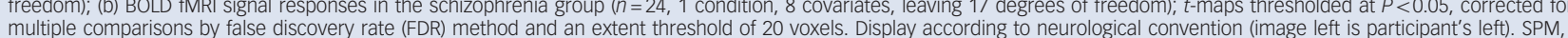
multiple comparisons by false
statistical parametric maps.

frontal gyrus and cerebellar declive. The brain regions that showed BOLD activations in both the control group as well as schizophrenia group are depicted in Fig. 3 (central area: A) and those brain regions that were activated only in the schizophrenia group are shown in the outer ring (B) of Fig. 3.

\section{BOLD deactivations associated with word generation in control and schizophrenia groups}

A one-sample random-effects analysis (contrast: word repetition $v$. word generation; false discovery rate $P<0.05$ with 20 voxels extent threshold) in the control group revealed widespread areas of increased BOLD signal in brain regions that were not activated during word generation (Fig. 4(a) and Fig. DS5, Table DS5). The schizophrenia group, however, did not reveal any areas of increased BOLD signal for this contrast, reflecting lack of deactivation of the above brain regions during word generation (Fig. 4(b)).

Figure DS6 shows the word generation $v$. word repetition and the word repetition $v$. word generation $t$-maps for the control and schizophrenia groups, surface-rendered on a single participant's canonical brain in the MNI space.

\section{Between-group comparisons of BOLD activations (schizophrenia > control group) and deactivations (control > schizophrenia group)}

Based on the results of the one-sample random-effects analyses, a post hoc between-group comparison of BOLD activations was carried out using SnPM to examine the brain regions that showed excessive activations in the schizophrenia group when compared with the control group (Fig. 5). The brain regions that showed higher activations in the schizophrenia group $(P<0.01$ uncorrected $)$ are given in Table DS6. Similarly, post hoc group comparisons were done to examine the brain regions that showed greater deactivations in the control group when compared with the schizophrenia group. The brain regions that showed greater deactivations in the control group $(P<0.01$, uncorrected $)$ are given in Table DS7.

\section{BOLD deactivations and response latencies}

The lat $\mathrm{gen}_{\text {gen }}-\mathrm{lat}_{\text {rep }}$ values computed from the available response latencies ( $n=42$ : control group $n=21$; schizophrenia group $n=21$ ) were significantly higher in those in the schizophrenia group in comparison with the control group (control group: mean $37.57 \mathrm{~ms}$ (s.d. =111.68); schizophrenia group: mean $131.29 \mathrm{~ms}$ (s.d.122.86); $t=2.59 ; P<0.013)$. The regression analysis was carried out on a subset of the above 42 participants $(n=31$ : control group $n=14$; schizophrenia group $n=17$ ), who had positive lat ${ }_{\text {gen }}-$ lat $_{\text {rep }}$ values. In this subset of participants as well, the lat $\mathrm{gen}_{\text {gen }}-$ lat $_{\text {rep }}$ values were significantly higher in those in the schizophrenia group in comparison with those in the control group (control group: mean $89.50 \mathrm{~ms}$ (s.d. $=93.31$ ); schizophrenia group mean $175.71 \mathrm{~ms}$ (s.d. $=87.17$ ); $t=2.66 ; P<0.013$ ). The control group showed a significant inverse relationship of task-related deactivation with lat gen - lat $_{\text {rep }}$ in broadly the same brain areas that were deactivated during word generation (Fig. 6, Table DS8), whereas no significant relationship was found between lat gen - lat $_{\text {rep }}$ and task-related activations $(P<0.05$, false discovery rate corrected with 20 voxels extent threshold). The schizophrenia group, however, failed to show any relationship between task-related activation/deactivation and lat ${ }_{\text {gen }}-$ lat $_{\text {rep. }}$.

\section{Discussion}

The present study provides fMRI evidence for inefficient activations and deficient deactivations underlying impaired verbal fluency in individuals with schizophrenia when compared with matched healthy controls. The inferences of the study were derived from the results of one-sample random-effects analyses, which permit us to make population-level inferences from the generated results. $^{23}$ Random-effects analysis takes into account withinparticipant variability as well as between-participant variability, thus permitting generalisability of inferences from multiparticipant functional neuroimaging to the population level. Interestingly, the brain regions recruited by the control group during word generation were replicated with minimal redundancies by the schizophrenia group. The schizophrenia 


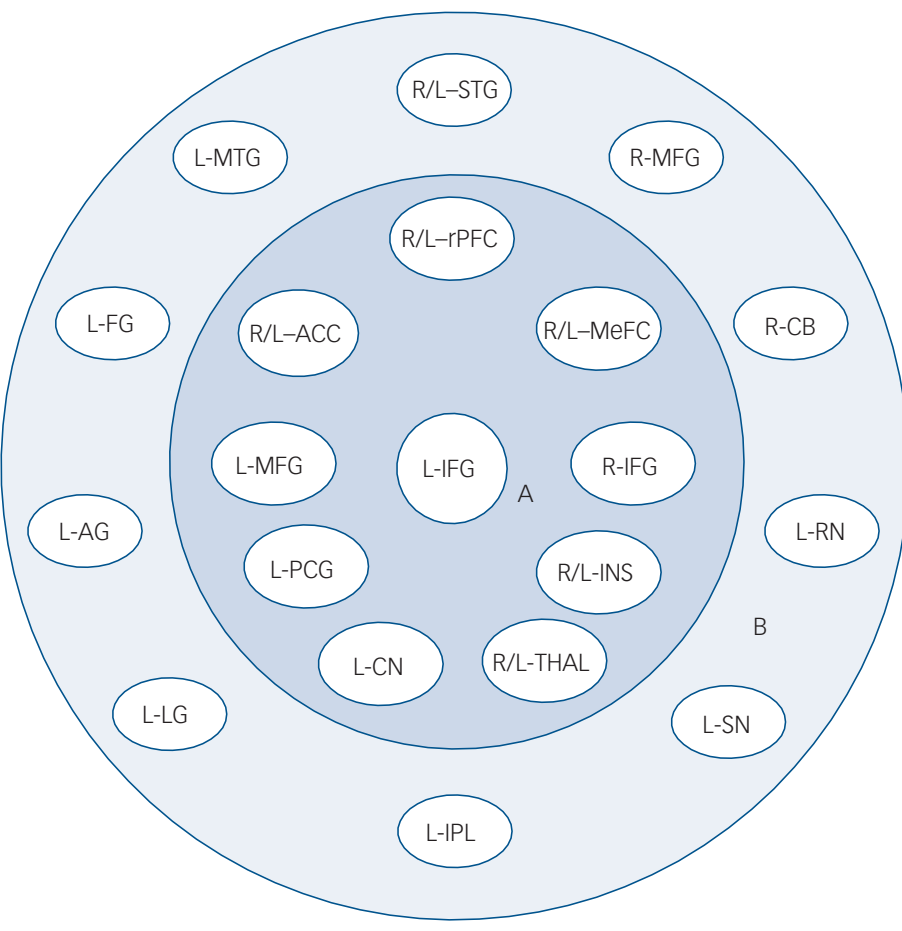

The central area (A) shows the core/essential brain regions and cognitive/associated processes underlying overt, semantic category word generation in healthy individuals

1. L-IFG - Semantic search, word retrieval and selection

2. R-IFG - Suppression of inappropriate responses

3. ACC - Control functions (e.g. selective attention: facilitation and suppression of within-category responses: task adherence; monitoring of conflicting responses and errors; recruiting essential brain regions)

4. MeFC - Higher-order motor control processes such as setting up goals of internal selection of individual actions (pre-SMA); motor preparation for oral expression (SMA)

5. rPFC - Executive control and maintenance of internally generated cognitive operations in word generation

6. INS - Sensory, motor and mental control of selection and suppression mechanisms

7. L-MFG - Updating generated words in verbal working memory

8. THAL - Speech production and articulation; generation of mental images and visual details

9. $\mathrm{CN}$ - speech production and articulation

10. L-PCG - Articulatory processes during production of verbal responses

\section{Fig. 3 Brain regions activated during overt semantic category word generation.}

Central area (A): core/essential brain areas commonly activated in both the control and schizophrenia groups. The left caudate nucleus ( $\mathrm{L}-\mathrm{CN}$ ) was the only exception, being activated in the control group but not in the schizophrenia group. Outer ring (B): additional brain areas recruited exclusively by the schizophrenia group. The cognitive/associate processes underlying overt, semantic category word generation, mediated by the essential brain regions, are enlisted. L-, left; R-, right; R/L, bilateral; IFG, inferior frontal gyrus; $\mathrm{ACC}$, anterior cingulate cortex; rPFC, rostral prefrontal cortex; MeFC, medial frontal cortex; MFG, middle frontal gyrus; INS, insula; CN, caudate nucleus; THAL, thalamus; CBL, cerebellum; STG, superior temporal gyrus; MTG, middle temporal gyrus; FG, fusiform gyruS; AG, angular gyrus; IPL, inferior parietal lobule; SN, substantia nigra; RN, red nucleus; SMA, supplementary motor area.

(a)
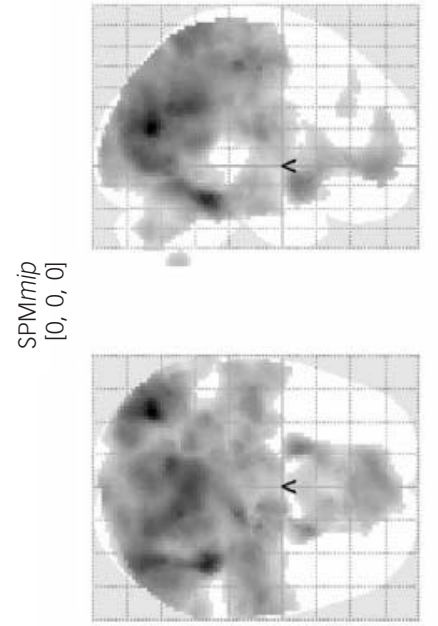

SPMresults: . Ios nc deact \results3

Height threshold $\mathrm{T}=2.253727\{P<0.05$ (FDR) Extent threshold $\mathrm{k}=20$ voxels

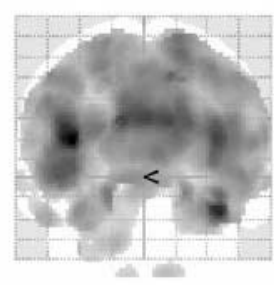

(b)

$\operatorname{SPM}\left[\mathrm{T}_{17}\right]$
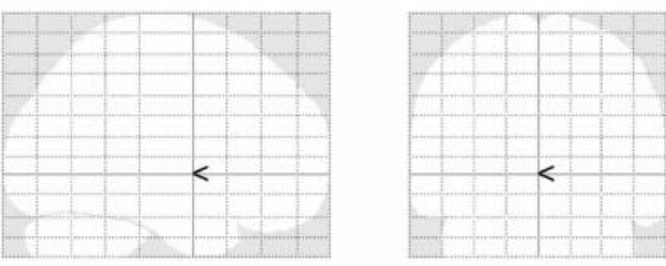

응ㅇ
$\sum_{0}^{0} 0^{\circ}$

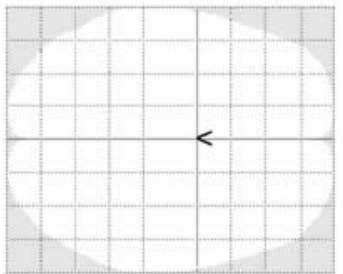

SPMresults: Ios sz deact\results5 Height threshold $\mathrm{T}=\operatorname{Inf}\{P<0.05(\mathrm{FDR})\}$ Extent threshold $k=0$ voxels

\section{Fig. 4 One-sample random-effects analysis, word repetition-word generation contrast.}

(a) Blood oxygen level dependent (BOLD) functional magnetic resonance imaging (fMRI) signal responses in control group ( $n=24,1$ condition, 6 covariates, leaving 17 degrees of freedom); (b) BOLD fMRI signal responses in schizophrenia group $(n=24,1$ condition, 8 covariates, leaving 17 degrees of freedom); $t$-maps thresholded at $P<0.05$, corrected for multiple comparisons by false discovery rate (FDR) method and an extent threshold of 20 voxels. Display according to neurological convention (image left is participant's left). SPM statistical parametric maps.

group showed more extensive activations as well as deficient deactivations of other brain regions during word generation, in contrast to the control group. Moreover, the results of the regression analysis provided confirmatory evidence for the relationship between cortical deactivation and behavioural performance during word generation in healthy individuals.

\section{Behavioural performance in the control and schizophrenia groups}

The participants with schizophrenia in the present study generated fewer overall responses as well as correct responses, while having a significantly larger number of 'no response' trials 
(a)
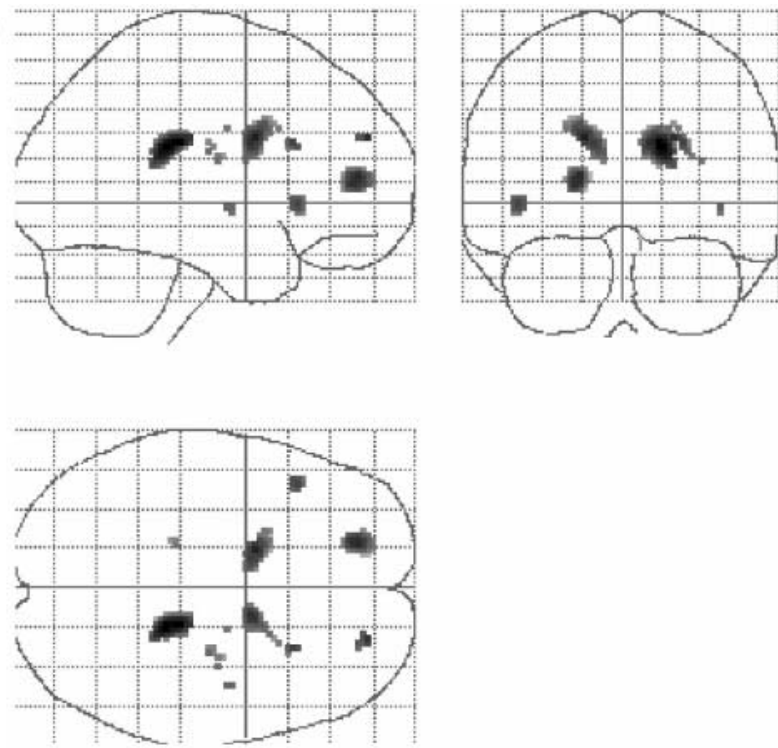

(c)
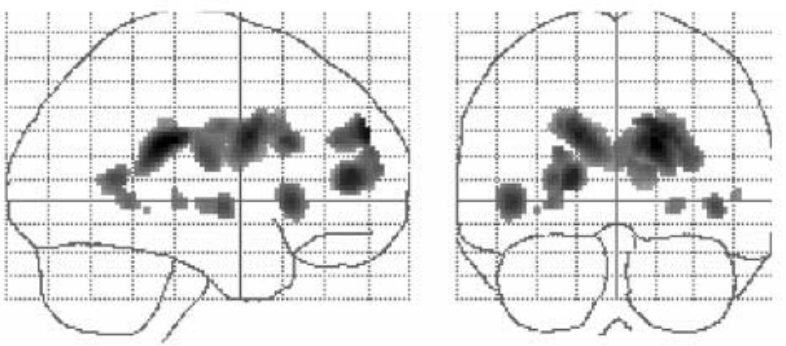

(b)
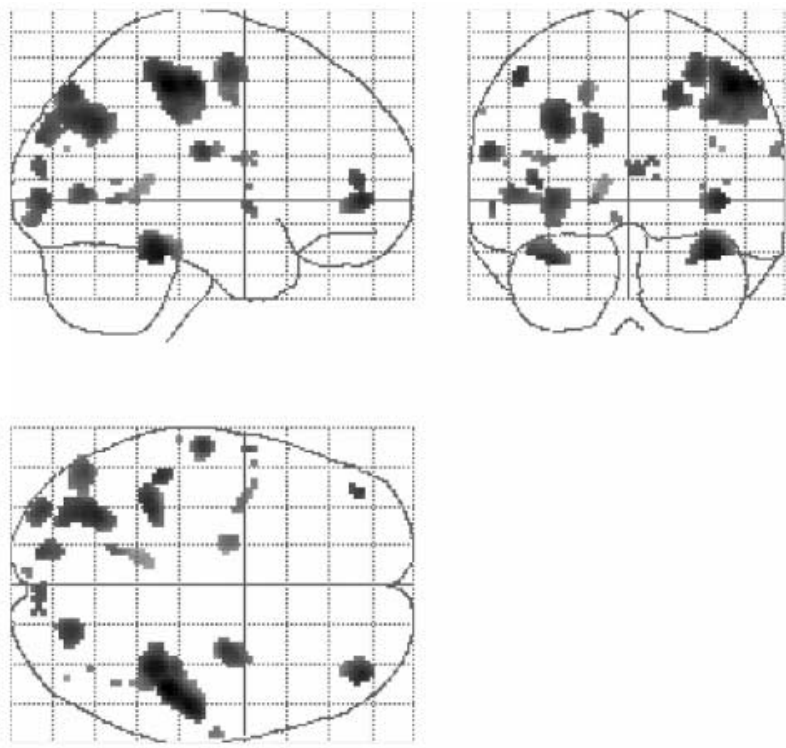

(d)
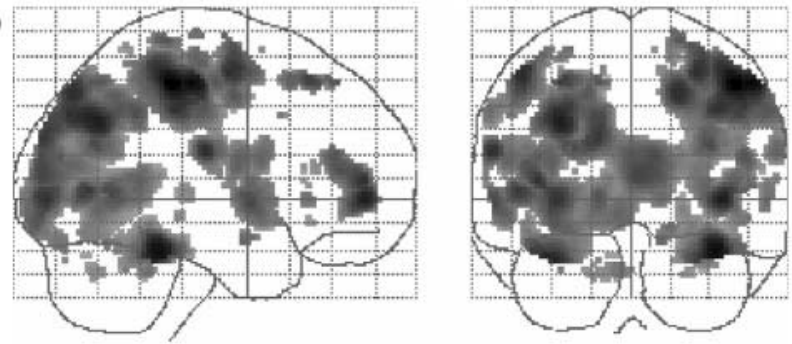
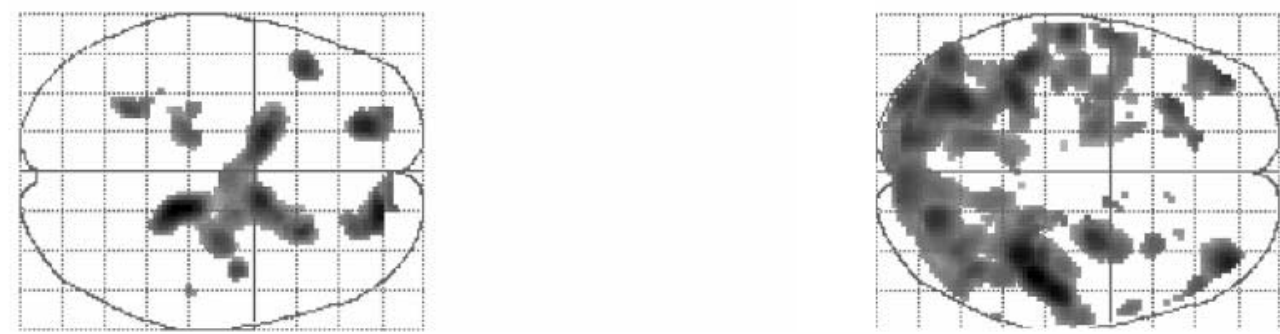

\section{Fig. 5 Results of non-parametric permutations-based two-sample pseudo $t$-test implemented in SnPM.}

The schizophrenia group $(n=24)$ showed greater activations than the control group $(n=24)$ at a significance threshold of $P<0.01$, uncorrected (a). At $P<0.05$ uncorrected, more brain regions showed greater activations in the schizophrenia group than the control group (c). Similarly, the control group showed greater deactivations than the schizophrenia group at a significance threshold of $P<0.01$ uncorrected (b), with more areas showing greater deactivation at the more liberal threshold of $P<0.05$ uncorrected (d). Display according to neurological convention (image left is participant's left).

(Table 2). The finding of poorer performance of participants with schizophrenia in the word generation trials is in line with the results of previous neuropsychological studies using standard verbal fluency task paradigms (for example Bozikas $e a^{24}$ ) as well as studies using standard fMRI activation paradigms (for example Boksman $e t a^{25}$ ). The schizophrenia group achieved a degree of accuracy in the word repetition trials (98.4\% correct responses) comparable to that of the control group (100\%).

\section{Brain activations during word generation in control and schizophrenia groups}

Previous studies that have used covert word generation tasks have reported predominantly left-sided activations; however, bilateral prefrontal activations have been reported by studies using overt paradigms. ${ }^{9}$ Therefore, the activations observed in the present study (Tables DS3 and DS4, Figs 2 and DS4) reflect bilateral involvement of brain regions underlying overt word generation. Additionally, it must be kept in mind that the greater cognitive and articulatory (greater complexity of the generated words $v$. 'pass') demands, over and above the core process of semantic word generation, may also have contributed towards the activations observed during the word generation condition in comparison with the word repetition condition (Fig. 3). The brain regions that were activated during word generation in the control group, as enumerated earlier, were replicated in the schizophrenia group with the exception of the left caudate nucleus and bilateral thalamus. These core/essential brain regions have been shown by previous research to be mediating the cognitive processes associated with semantic category word generation. ${ }^{8,9,26-37}$ 

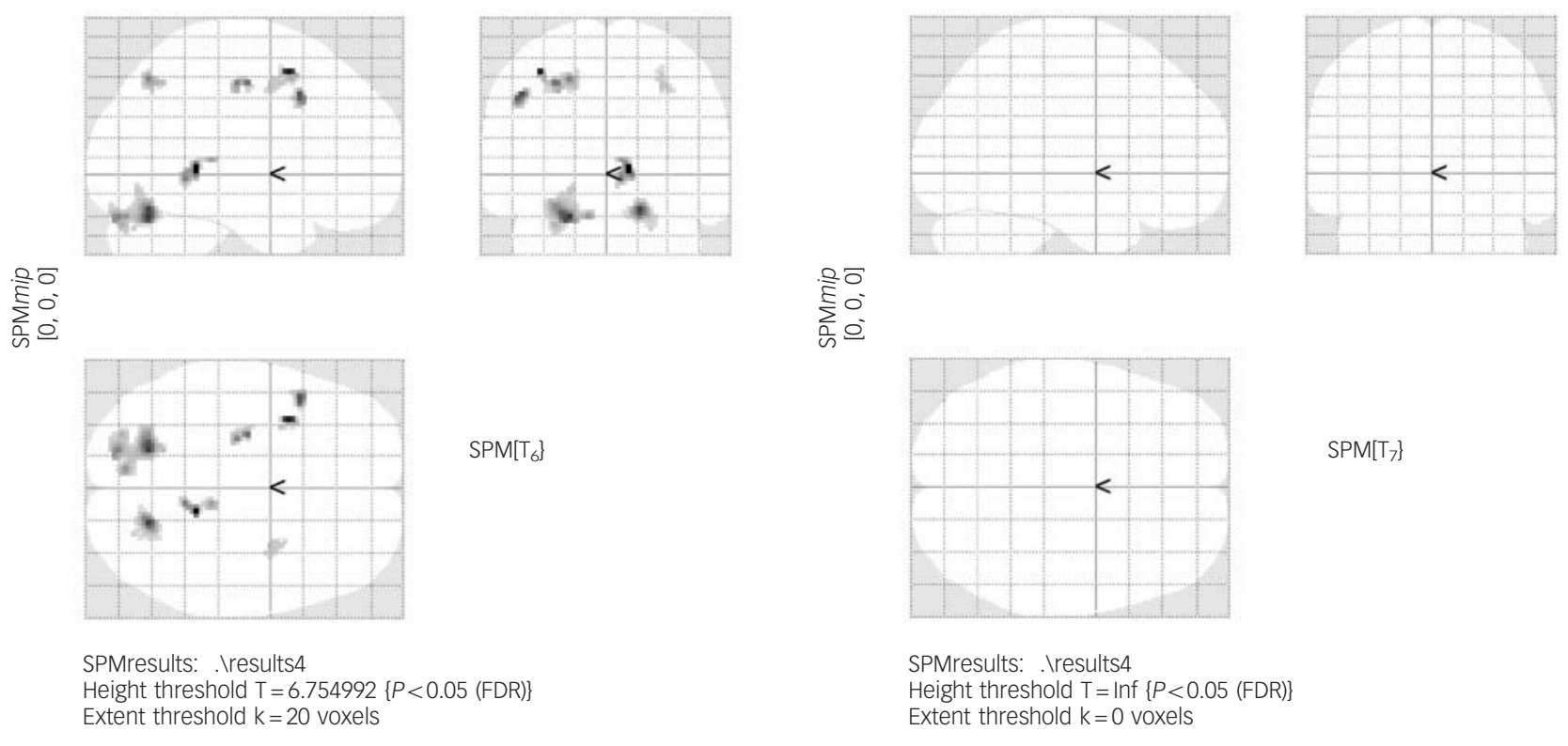

Fig. 6 Regression analysis: word generation-word repetition contrast images regressed with difference of word generation-word repetition latencies.

(a) control group; (b) schizophrenia group. $P<0.05$, corrected for multiple comparisons by false discovery rate (FDR) method and an extent threshold of 20 voxels. Display according to neurological convention (image left is participant's left). SPM, statistical parametric maps.

The schizophrenia group showed additional activations of multiple cortical regions as well as red nucleus and cerebellar declive during word generation, despite significantly poorer behavioural performances compared with the control group (Table 4, Figs 2(b) and DS4). These brain regions have also been shown to subserve cognitive processes involved in word generation $^{8,9,28,29,34-36,38}$ and are therefore presumably recruited even by healthy individuals during word generation; however, the fact that these were shown to be more active only in the schizophrenia group reflects greater activations of the above regions during effortful performance of word generation by the cognitively compromised participants with schizophrenia. ${ }^{10}$ Previous studies in individuals with language dysfunction due to coarse brain disease have reported higher prefrontal activations in comparison with healthy controls; ${ }^{39}$ therefore, it may be inferred that higher activations during the performance of cognitive functions need not necessarily indicate better performance, but, on the contrary, may indicate compromised functional ability. Our finding of this 'inefficient' fMRI BOLD response in the schizophrenia group during word generation is in concordance with those of the only previous study that used a similar paradigm. ${ }^{10}$

It is interesting to note that the core brain regions reported to be associated with word generation in both healthy individuals and those with schizophrenia from Western settings were indeed replicated in the present study, which to the best of our knowledge is the first of its kind from a non-Western setting. Thus, it may be inferred that the neural substrates of word generation are common despite cultural and linguistic differences. Specifically, previous studies from the West (for example Bookheimer, ${ }^{26}$ Costafreda et $a l^{27}$ ) have demonstrated a functional specialisation within the left inferior frontal gyrus, with the anterior left inferior frontal gyrus (Brodmann area 47/46) being important for semantic category word generation as well as executive processes of semantic processing, whereas the posterior inferior frontal gyrus (Brodmann area 44) appears to be specialised for phonological and syntactic processing. In the present study, which used a semantic word generation paradigm, the Brodmann area 47/46, and not Brodmann area 44, was activated during word generation in both the control and schizophrenia groups (Tables DS3 and DS4). As mentioned above, our finding of more extensive cortical activations in participants with schizophrenia during word generation is in agreement with the only previous study from the West ${ }^{10}$ that employed a similar fMRI BOLD acquisition paradigm. Additionally, other studies from the West (for example Frith et $a l,{ }^{40}$ Yurgelun-Todd et $a l,{ }^{41}$ Liddle $^{42}$ ) have reported increased superior temporal gyrus activity in participants with schizophrenia relative to healthy comparison participants. This might indicate that the structural/functional basis of word generation is similar across languages and that the excessive cortical activations accompanying impaired verbal fluency in schizophrenia may also be similar across languages/cultures.

\section{Brain deactivations during word generation in the control and schizophrenia groups}

The word repetition $v$. word generation one-sample randomeffects contrast revealed extensive signals in healthy participants in regions that were not primarily involved in word generation (Figs 4(a) and DS5, Table DS5), whereas the lack of apparent BOLD differences in the word repetition $v$. word generation contrast in the schizophrenia group (Fig. 4(b)) may be interpreted to reflect their inability to 'deactivate' the above regions, as detailed in the introduction.

Additional confirmatory evidence highlighting the significance of task-related deactivation for behavioural performance was provided by the results of our regression analyses, which revealed a significant inverse relationship between the extent of deactivations and the difference in response latencies between word generation and word repetition trials $\left(\operatorname{lat}_{\text {gen }}-\right.$ lat $_{\text {rep }}$ ) in the control group (Fig. 6, Table DS8). Interestingly, no such relationship between task-related deactivation and lat ${ }_{\text {gen }}-$ lat $_{\text {rep }}$ was found in the schizophrenia group. The absence of a significant relationship between behavioural performance and task-related deactivation in the schizophrenia group may be explained by 
the fact that participants with schizophrenia as a group showed deficient deactivations irrespective of the level of performance, as revealed by our one-sample random-effects analyses.

\section{Task-related excessive activations and deficient deactivations in schizophrenia}

Post hoc between-group comparisons using SnPM revealed excessive activations in the schizophrenia group during word generation in bilateral caudate nuclei and rostral prefrontal cortices (Brodmann area 10); inferior frontal gyrus (Brodmann area 47 ) on the left side; and insula (Brodmann area 13), cingulate gyrus (Brodmann area 24 and 32) and claustrum on the right side $(P<0.01$ uncorrected) (Table DS6). Previous fMRI studies of word generation employing different experimental protocols have shown excessive activations in individuals with schizophrenia and/ or those at high risk for schizophrenia of the left inferior frontal gyrus; ${ }^{10,18}$ right inferior frontal gyrus; ${ }^{43}$ right anterior cingulate cortex, ${ }^{10}$ as well as other regions, including superior temporal and parietal cortices and subcortical structures. ${ }^{10,40-42}$ The post hoc between-group comparisons using SnPM also revealed higher deactivations in the control group in multiple brain regions (Table DS7), when compared with the schizophrenia group. To the best of our knowledge, there have been no previous reports of fMRI studies that were optimally designed to detect deactivations of brain regions during word generation.

The above finding in people with schizophrenia of inadequate deactivations of brain regions that do not constitute core/essential areas involved in word generation, leading possibly onto a more diffuse and an inefficient neuronal response, could constitute the neurophysiological signature of the 'defective cognitive filter' ${ }^{44}$ in schizophrenia. We therefore propose that inadequate deactivations of competing brain regions and inability to inhibit the cognitive processes that they subserve could constitute the fundamental information-processing deficit underlying impaired word generation in schizophrenia.

The aberrant activations and deactivations observed in schizophrenia during word generation may also be heuristically linked to the proposed neurotransmitter dysfunctions underlying schizophrenia pathophysiology. The core neurobiological dysfunction underlying schizophrenia has been proposed to be the result of $N$-methyl-D-aspartate (NMDA) receptor hypofunction ${ }^{45}$ in the cortical GABA-ergic interneurons, ${ }^{46}$ as well as striatal hyperdopaminergia, ${ }^{47}$ which in turn would result in a net cortical excitatory state. The net cortical excitation secondary to NMDA hypofunction has been shown to be reflected in the fMRI BOLD response as task-related excessive activations and/or deficient deactivations. ${ }^{48,49}$ Interestingly, greater task-related activations in the inferior frontal cortex during the performance of a verbal fluency task in people at high risk for schizophrenia were found to be directly correlated with striatal dopamine levels in a recent multi-modal imaging study using fMRI and ${ }^{18} \mathrm{~F}$-dopa positron emission tomography (PET). ${ }^{18}$ Therefore, the findings of our study provide indirect support to the cortical NMDA hypofunction as well as striatal hyperdopaminergic hypotheses of schizophrenia. However, future hypothesis-driven studies are required to confirm the link between NMDA-receptor hypofunction as well as elevated striatal dopaminergic function and aberrant fMRI BOLD haemodynamic responses in schizophrenia.

\section{Limitations}

The gender distribution of the study samples was not equal (Table $1)$, but the difference was not statistically significant $\left(\chi^{2}=0.95\right.$, $P<0.33)$. Moreover, re-analysis excluding female participants from both groups revealed similar results as that of the overall sample. Additionally, gender was used as a covariate in all the analyses. In total 15 of the 24 participants with schizophrenia were receiving antipsychotic and/or anticholinergic medications at the time of fMRI acquisition; this could have potentially increased cerebral blood flow to the prefrontal cortex ${ }^{17,18}$ and the basal ganglia. ${ }^{19}$ To avoid the confounding effects of medications, we included two regressors (mean risperidone equivalent dosage and mean trihexyphenidyl dosage) in the random-effects analyses. Dopamine receptors $\left(\mathrm{D}_{2}\right)$ receptor occupancy with regular clinical doses of risperidone and olanzapine were found to be similar; however, clozapine showed comparatively much lower $\mathrm{D}_{2}$ occupancies in clinically effective doses, ${ }^{50}$ suggesting the contribution of non- $\mathrm{D}_{2}$ receptors such as $5-\mathrm{HT}_{2}$ to antipsychotic effects. Therefore the antipsychotic doses expressed as risperidone equivalents may be considered as only surrogate indices for antipsychotic-induced blood-flow variability mediated by their actions on multiple target receptors. Nevertheless, it may be pointed out that 9 of the participants with schizophrenia out of the sample of 24 were antipsychotic-naive/free and the remaining 15 were on a mean risperidone dose of $4.92 \mathrm{mg}$ per day (Table 1 ). One-sample random-effects analyses confirmed the pattern of excessive activations and deficient deactivations in participants with schizophrenia during word generation at a conservative significance threshold $(P<0.05$, false discovery rate corrected). However, post hoc two-sample between-group comparisons using SnPM revealed group differences only at a more liberal threshold $(P<0.01$, uncorrected $)$, apparently as a result of the lower power afforded by the non-parametric analysis, given the heterogeneity in the covariance structure between the schizophrenia group and the control group. However, it may be pointed out that the between-group comparisons were carried out only as a post hoc analysis in order to support the primary results of the study, as detailed earlier. Moreover, the two-sample results mirrored the one-sample results of excessive activations and inadequate deactivations in participants with schizophrenia, albeit at a lower significance threshold. The results of the regression analysis confirmed the relationship between improved behavioural performance during word generation and the greater extent of task-related deactivation in the control group alone and not in the schizophrenia group. This may have been because of the fact that the participants with schizophrenia as a group showed inefficient activations and deficient deactivations irrespective of performance, as discussed above.

John P. John, MD, Harsha N. Halahalli, MD, MPhil, Department of Psychiatry, National Institute of Mental Health and Neurosciences, Bangalore; Mandapati K. Vasudev, MD (deceased); Peruvumba N. Jayakumar, MD, Department of Neuroradiology and Interventional Radiology, National Institute of Mental Health and Neurosciences, Bangalore; Sanjeev Jain, MD, Department of Psychiatry, National Institute of Mental Health and Neurosciences, Bangalore, India.

Correspondence: John P. John, MD, Associate Professor of Psychiatry, National Institute of Mental Health and Neurosciences (NIMHANS), Post Box Number 2900, Dharmaram Post Office, Hosur Road, Bangalore, 560 029, Karnataka, India. Email: jpj@nimhans.kar.nic.in; jpjnimhans@gmail.com

First received 10 Jun 2010, final revision 12 Oct 2010, accepted 15 Dec 2010

\section{Funding}

This study was supported by the Department of Biotechnology (DBT), Government of India (Grant No. BT/PR/8363/MED/14/1252 to J.P.J.).

\section{Acknowledgements}

Professor Mandapati K. Vasudev, Head of Department of Neuroradiology and Interventional Radiology (NIIR) at NIMHANS, sadly passed away on the 16 September 2010. The authors Radiology (NIIR) at NIMHANS, sadly passed away on the 16 September 2010. The authors
acknowledge with gratitude, the contributions of Professor Vasudev in facilitating the acquisition of fMRIs for the study as well as in the preparation of the manuscript for publication. 
We thank Mr Kiran Kumar for his assistance in image acquisition and Dr Vikram Arunachalam and Dr Abish Antony for carrying out recruitment of participants and psychopathology ratings.

\section{References}

1 Szöke A, Trandafir A, Dupont M-E, Méary A, Schürhoff F, Leboyer M. Longitudinal studies of cognition in schizophrenia: meta-analysis. Br J Psychiatry 2008; 192: 248-57.

2 Fu CHY, Suckling J, Williams SCR, Andrew CM, Vythelingum GN, McGuire PK. Effects of psychotic state and task demand on prefrontal function in schizophrenia: an fMRI study of overt verbal fluency. Am J Psychiatry 2005 162: $485-94$.

3 Schlösser R, Hutchinson M, Joseffer S, Rusinek H, Saarimaki A, Stevenson J, et al. Functional magnetic resonance imaging of human brain activity in a verbal fluency task. J Neurol Neurosurg Psychiatr 1998; 64: 492-8.

4 Indefrey $\mathrm{P}$, Levelt WJM. The neural correlates of language production. In The New Cognitive Neurosciences (ed M Gazzaniga): 845-65. MIT Press, 2000.

5 Cabeza R, Nyberg L. Imaging cognition II: an empirical review of 275 PET and fMRI studies. J Cogn Neurosci 2000; 12: 1-47.

6 Melinder MRD, Barch DM, Heydebrand G, Csernansky JG. Easier tasks can have better discriminating power: the case of verbal fluency. J Abnorm Psychol 2005; 114: 385-91.

7 Basho S, Palmer ED, Rubio MA, Wulfeck B, Müller R. Effects of generation mode in fMRI adaptations of semantic fluency: paced production and over speech. Neuropsychologia 2007; 45: 1697-706.

8 Fu CHY, Morgan K, Suckling J, Williams SCR, Andrew C, Vythelingum GN, et al. A functional magnetic resonance imaging study of overt letter verbal fluency using a clustered acquisition sequence: greater anterior cingulate activation with increased task demand. Neuroimage 2002; 17: 871-9.

9 Abrahams S, Goldstein LH, Simmons A, Brammer MJ, Williams SCR, Giampietro VP, et al. Functional magnetic resonance imaging of verbal fluency and confrontation naming using compressed image acquisition to permit overt responses. Hum Brain Mapp 2003; 20: 29-40.

10 Ragland JD, Moelter ST, Bhati MT, Valdez JN, Kohler CG, siegel SJ, et al. Effect of retrieval effort and switching demand on fMRI activation during semantic word generation in schizophrenia. Schizophr Res 2008; 99: 312-23.

11 Annett M. A coordination of hand preference and skill replicated. Br J Psychol 1976; 67: 587-92.

12 Folstein MF, Folstein SE, McHugh PR. "Mini-mental state". A practical method for grading the cognitive state of patients for the clinician. J Psychiatr Res 1975; 12: 189-98.

13 Wechsler D. WAIS III Administration and Scoring Manual. Psychological Corporation, 1997

14 American Psychiatric Association. Diagnostic and Statistical Manual of Mental Disorder (4th edn) (DSM-IV). APA, 1994

15 Sheehan DV, Lecrubier $Y$, Sheehan $\mathrm{KH}$, Amorim $\mathrm{P}$, Janavs J, Weiller $\mathrm{E}$, et al The Mini-International Neuropsychiatric Interview (M.I.N.I.): the development and validation of a structured diagnostic psychiatric interview for DSM-IV and ICD-10. J Clin Psychiatry 1998; 59 (suppl 20): 22-33.

16 Woods SW. Chlorpromazine equivalent doses for the newer atypical antipsychotics. J Clin PSychiatry 2003; 64: 663-7.

17 Honey GD, Bullmore ET, Soni W, Varatheesan M, Williams SC, Sharma T. Differences in frontal cortical activation by a working memory task after substitution of risperidone for typical antipsychotic drugs in patients with schizophrenia. Proc Natl Acad Sci USA 1999; 96: 13432-7.

18 Fusar-Poli $\mathrm{P}$, Howes OD, Allen P, Broome M, Valli I, Asselin M, et al. Abnormal prefrontal activation directly related to pre-synaptic striatal dopamine dysfunction in people at clinical high risk for psychosis. $\mathrm{Mol}$ Psychiatry 2011; 16: 67-75.

19 Miller DD, Andreasen NC, O'Leary DS, Rezai K, Watkins GL, Ponto LL, et al. Effect of antipsychotics on regional cerebral blood flow measured with positron emission tomography. Neuropsychopharmacology 1997; 17: 230-40.

20 Kay SR, Fiszbein A, Opler LA. The positive and negative syndrome scale (PANSS) for schizophrenia. Schizophr Bull 1987; 13: 261-76.

21 Nichols TE, Holmes AP. Nonparametric permutation tests for functional neuroimaging: a primer with examples. Hum Brain Mapp 2002; 15: 1-25.

22 Lancaster JL, Woldorff MG, Parsons LM, Liotti M, Freitas CS, Rainey L, et al. Automated Talairach atlas labels for functional brain mapping. Hum Brain Mapp 2000; 10: 120-31.

23 Penny W, Holmes A. Random effects analysis. In Statistical Parametric Mapping: The Analysis of Functional Brain Images (eds K Friston, J Ashburner, S Kiebel, T Nichols, W Penny). Academic Press, 2007.

24 Bozikas VP, Kosmidis MH, Karavatos A. Disproportionate impairment in semantic verbal fluency in schizophrenia: differential deficit in clustering. Schizophr Res 2005; 74: 51-9.
25 Boksman K, Théberge J, Williamson $P$, Drost DJ, Malla A, Densmore $M$, et al. A 4.0-T fMRI study of brain connectivity during word fluency in first-episode schizophrenia. Schizophr Res 2005; 75: 247-63.

26 Bookheimer S. Functional MRI of language: new approaches to understanding the cortical organization of semantic processing. Annu Rev Neurosci 2002; 25: 151-88.

27 Costafreda SG, Fu CHY, Lee L, Everitt B, Brammer MJ, David AS. A systematic review and quantitative appraisal of fMRI studies of verbal fluency: role of the left inferior frontal gyrus. Hum Brain Mapp 2006; 27: 799-810.

28 Whitney C, Weis S, Krings T, Huber W, Grossman M, Kircher T. Task-dependent modulations of prefrontal and hippocampal activity during intrinsic word production. J Cogn Neurosci 2009; 21: 697-712.

29 Crosson B, Benefield H, Cato MA, Sadek JR, Moore AB, wierenga CE, et al. Left and right basal ganglia and frontal activity during language generation: contributions to lexical, semantic, and phonological processes. J Int Neuropsychol Soc 2003; 9: 1061-77.

30 Collette F, Van der Linden M, Delfiore G, Degueldre C, Luxen A, Salmon E. The functional anatomy of inhibition processes investigated with the Hayling task. Neuroimage 2001; 14: 258-67.

31 Barch DM, Braver TS, Sabb FW, Noll DC. Anterior cingulate and the monitoring of response conflict: evidence from an fMRI study of overt verb generation. J Cogn Neurosci 2000; 12: 298-309.

32 Burgess PW, Dumontheil I, Gilbert SJ. The gateway hypothesis of rostral prefrontal cortex (area 10) function. Trends Cogn Sci 2007; 11: 290-8.

33 Picard N, Strick PL. Motor areas of the medial wall: a review of their location and functional activation. Cereb Cortex 1996; 6: 342-53.

34 Gardini S, Cornoldi C, De Beni R, Venneri A. Cognitive and neuronal processes involved in sequential generation of general and specific mental images. Psychol Res 2009; 73: 633-43.

35 Gabrieli JD, Poldrack RA, Desmond JE. The role of left prefrontal cortex in language and memory. Proc Natl Acad Sci USA 1998; 95: 906-13.

36 Gaillard WD, Sachs BC, Whitnah JR, Ahmad Z, Balsamo LM, Petrella JR, et al. Developmental aspects of language processing: fMRI of verbal fluency in children and adults. Hum Brain Mapp 2003; 18: 176-85.

37 Price CJ, Moore CJ, Humphreys GW, Frackowiak RS, Friston KJ. The neural regions sustaining object recognition and naming. Proc Biol SCi 1996; 263: 1501-7.

38 Middleton FA, Strick PL. Anatomical evidence for cerebellar and basal ganglia involvement in higher cognitive function. Science 1994; 266: 458-61.

39 Bonner-Jackson A, Haut K, Csernansky JG, Barch DM. The influence of encoding strategy on episodic memory and cortical activity in schizophrenia. Biol Psychiatry 2005; 58: 47-55.

40 Frith $C D$, Friston KJ, Herold S, Silbersweig D, Fletcher P, Cahill C, et al. Regional brain activity in chronic schizophrenic patients during the performance of a verbal fluency task. Br J Psychiatry 1995; 167: 343-9.

41 Yurgelun-Todd DA, Waternaux CM, Cohen BM, Gruber SA, English CD, Renshaw PF. Functional magnetic resonance imaging of schizophrenic patients and comparison subjects during word production. Am J Psychiatry 1996; 153: 200-5.

42 Liddle PF. Functional imaging - schizophrenia. Br Med Bull 1996; 52: 486-94.

43 Weiss EM, Hofer A, Golaszewski S, Siedentopf C, Brinkhoff C, Kremser C, et al. Brain activation patterns during a verbal fluency test: a functional MRI study in healthy volunteers and patients with schizophrenia. Schizophr Res 2004; 70: 287-91.

44 Frith $C D$. Consciousness, information processing and schizophrenia. Br J Psychiatry 1979; 134: 225-35.

45 Carlsson A, Waters N, Holm-Waters S, Tedroff J, Nilsson M, Carlsson ML. Interactions between monoamines, glutamate, and GABA in schizophrenia: new evidence. Annu Rev Pharmacol Toxicol 2001; 41: 237-60.

46 Gordon JA. Testing the glutamate hypothesis of schizophrenia. Nat Neurosci 2010; 13: 2-4.

47 Howes OD, Montgomery AJ, Asselin M, Murray RM, Valli I, Tabraham P, et al. Elevated striatal dopamine function linked to prodromal signs of schizophrenia. Arch Gen Psychiatry 2009; 66: 13-20.

48 Breier A, Malhotra AK, Pinals DA, Weisenfeld NI, Pickar D. Association of ketamine-induced psychosis with focal activation of the prefrontal cortex in healthy volunteers. Am J Psychiatry 1997; 154: 805-11.

49 Vollenweider FX, Leenders KL, Scharfetter C, Antonini A, Maguire P, Missimer $\mathrm{J}$, et al. Metabolic hyperfrontality and psychopathology in the ketamine model of psychosis using positron emission tomography (PET) and [18F]fluorodeoxyglucose (FDG). Eur Neuropsychopharmacol 1997; 7: 9-24.

50 Kapur S, Zipursky RB, Remington G. Clinical and theoretical implications of 5-HT2 and D2 receptor occupancy of clozapine, risperidone, and olanzapine in schizophrenia. Am J Psychiatry 1999; 156: 286-93. 